INPLASY

PROTOCOL

To cite: Kungwankiattichai et al. Maintenance with Hypomethylating Agents after Allogeneic Stem Cell Transplantation in Acute Myeloid Leukemia and Myelodysplastic Syndrome: A Systematic Review and MetaAnalysis. Inplasy protocol 2021110078. doi:

10.37766/inplasy2021.11.0078

Received: 21 November 2021

Published: 21 November 2021

Corresponding author: Weerapat Owattanapanich

weerapato36733@gmail.com

Author Affiliation:

Division of Hematology, Department of Medicine, Faculty of Medicine Siriraj Hospital, Mahidol University, Bangkok, Thailand.

Support: None.

Review Stage at time of this submission: Data analysis Completed but not published.

Conflicts of interest:

None declared.

\section{Maintenance with Hypomethylating Agents after Allogeneic Stem Cell Transplantation in Acute Myeloid Leukemia and Myelodysplastic Syndrome: A Systematic Review and Meta-Analysis}

Kungwankiattichai, S1; Ponvilawan, B2; Roy, C3; Tunsing, P4; Kuchenbauer, F5; Owattanapanich, W6.

Review question / Objective: P: Patients with AML or MDS after allo-SCT; I: Hypomethylating agents after allo-SCT; C: Observation after allo-SCT; O: Overall survival rates.

Condition being studied: Hypomethylating agents (HMAs) seem to have a range of properties favorable to postallogeneic hematopoietic stem cell transplantation (allo-SCT) maintenance in acute myeloid leukemia (AML) patients. This meta-analysis was performed to review all relevant studies to compare the outcomes of patients undergoing allo-SCT for AML or MDS receiving HMA maintenance therapy with observation only.

Information sources: The systematic search of the Embase and MEDLINE databases identified 4,416 articles, from which 512 duplicates were removed. This resulted in 3,904 articles available for title and abstract review. Subsequently, 3,875 articles were excluded as the article type and study design did not fulfill the inclusion criteria, or there was no report on a primary outcome of interest. The remaining 29 articles underwent full-length review and 18 of those were excluded for the aforementioned reasons. Ultimately, the eligibility criteria for our meta-analysis were met by 11 studies: 2 RCTs, 1 prospective cohort study, and 8 retrospective cohort studies.

INPLASY registration number: This protocol was registered with the International Platform of Registered Systematic Review and Meta-Analysis Protocols (INPLASY) on 21 November 2021 and was last updated on 21 November 2021 (registration number INPLASY2021110078).

\section{INTRODUCTION}

Review question / Objective: P: Patients with AML or MDS after allo-SCT; I:
Hypomethylating agents after allo-SCT; C: Observation after allo-SCT; $O$ : Overall survival rates. 
Condition being studied: Hypomethylating agents (HMAs) seem to have a range of properties favorable to post-allogeneic hematopoietic stem cell transplantation (allo-SCT) maintenance in acute myeloid leukemia (AML) patients. This metaanalysis was performed to review all relevant studies to compare the outcomes of patients undergoing allo-SCT for AML or MDS receiving HMA maintenance therapy with observation only.

\section{METHODS}

Search strategy: The systematic search of the Embase and MEDLINE databases identified 4,416 articles, from which 512 duplicates were removed. This resulted in 3,904 articles available for title and abstract review. Subsequently, 3,875 articles were excluded as the article type and study design did not fulfill the inclusion criteria, or there was no report on a primary outcome of interest. The remaining 29 articles underwent full-length review and 18 of those were excluded for the aforementioned reasons. Ultimately, the eligibility criteria for our meta-analysis were met by 11 studies: 2 RCTs, 1 prospective cohort study, and 8 retrospective cohort studies. Nine of these compared azacitidine maintenance to observation, whereas 2 compared decitabine maintenance to observation.

Participant or population: Patients underwent allo-SCT for AML or MDS.

Intervention: Hypomethylating agents maintenance after allo-SCT.

\section{Comparator: Observation.}

Study designs to be included: Studies had to be either randomized controlled trials (RCTs) or cohort studies (prospective or retrospective).

Eligibility criteria: The patients underwent allo-SCT for AML or MDS; (3) the studies compared 2 patient groups: one receiving an HMA post-allo-SCT, and the other being an observational group; and (4) the studies needed to report at least one of our primary outcomes of interest (OS, RFS, grades II-IV acute GVHD, and chronic GVHD).

Information sources: The systematic search of the Embase and MEDLINE databases identified 4,416 articles, from which 512 duplicates were removed. This resulted in 3,904 articles available for title and abstract review. Subsequently, 3,875 articles were excluded as the article type and study design did not fulfill the inclusion criteria, or there was no report on a primary outcome of interest. The remaining 29 articles underwent full-length review and 18 of those were excluded for the aforementioned reasons. Ultimately, the eligibility criteria for our meta-analysis were met by 11 studies: 2 RCTs, 1 prospective cohort study, and 8 retrospective cohort studies.

Main outcome(s): Primary outcomes - 1. overall survival (OS); 2 . relapse free survival (RFS); 3. grades II-IV acute GVHD; 4. chronic GVHD.

Additional outcome(s): Secondary outcomes - 1. cumulative incidence of relapse (CIR); 2. non-relapse mortality (NRM).

Quality assessment / Risk of bias analysis: The quality of each study using the Jadad scale for RCTs and the Newcastle-Ottawa scale for cohort studies

Strategy of data synthesis: Two investigators utilized a standardized collection form to extract the baseline characteristic data of the patients in each group, along with details of the primary and secondary outcomes of interest. The extracted data was cross-checked to confirm its accuracy.

Subgroup analysis: 1.Subgroup Analysis Based on Each HMA; 2.Subgroup Analysis of Patients Who Received HMAs in Combination with DLI.

Sensitivity analysis: We did not perform the sensitivity analysis. 
Language: English language.

Country(ies) involved: Thailand, Canada.

Keywords: acute myeloid leukemia; azacitidine; decitabine; hypomethylating agent; maintenance; transplant.

Contributions of each author:

Author 1 - Smith Kungwankiattichai - S.K. drafted the manuscript and prepared the final version.

Email: smithkung@yahoo.com

Author 2 - Ben Ponvilawa - B.P. collected the data. B.P. performed the statistical analyses.

Email: ben.ponv@gmail.com

Author 3 - Claudie Roy - C.R. designed the studies and approved the manuscript.

Email: claudie.roy@bccancer.bc.ca

Author 4 - Pattaraporn Tunsing - P.S. designed the studies and approved the manuscript.

Email: sakorn.tun@mahidol.ac.th

Author 5 - Florian Kuchenbauer - F.K. made critical revisions.

Email: fkuchenbauer@bccrc.ca

Author 6 - Weerapat Owattanapanich W.O. collected the data. W.O. drafted the manuscript and prepared the final version.

Email: weerapato36733@gmail.com 